Vasile Ene*, Ovidius University Constanţa, Romania Current address: 23 August 8717, Jud. Constanţa, Romania

e-mail:ene@s23aug.sfos.ro or ene@univ-ovidius.ro

\title{
ON THE DECOMPOSITION THEOREMS OF LEBESGUE AND JORDAN
}

\begin{abstract}
In the first part of this paper we show a powerful special case of Lebesgue's decomposition theorem, namely: if $F$ is a $V B$ function, satisfying Lusin's condition $(N)$ on $[a, b]$, then $F(x)-F(a)=s_{F}(x)+$ $(\mathcal{L}) \int_{a}^{x} F^{\prime}(t) d t$, where $s_{F}$ is the saltus function of $F$. In the second part we show that if $F$ satisfies Lusin's condition $(N)$ on $[a, b]$ then the functions (from the decomposition theorem of Jordan) $V_{F}(x):=V(F ;[a, x])$ and $G(x):=F(x)-V_{F}(x)$ also satisfy $(N)$.
\end{abstract}

The following decomposition theorem of Lebesgue is well known:

Theorem A (Lebesgue's decomposition theorem). ([7], p. 119).

If $F$ is an additive function of bounded variation of an interval, the derivative $F^{\prime}$ is summable, and the function $F$ is the sum of a singular additive function of an interval and of the indefinite integral of the derivative $F^{\prime}$.

Moreover, if the function $F$ is non-negative, we have for every interval $I_{o}$

$$
F\left(I_{o}\right) \geq \int_{I_{o}} F^{\prime}(t) d t,
$$

equality holding only in the case in which the function $F$ is absolutely continuous on $I_{o}$.

In the first part of this paper, for the special case of a function defined on an interval $[a, b]$, with bounded variation and satisfying Lusin's condition $(N)$, Theorem A becomes

$$
F(x)-F(a)=s_{F}(x)+(\mathcal{L}) \int_{a}^{x} F^{\prime}(t) d t,
$$

Key Words: The decomposition theorems of Lebesgue and Jordan, $A C, V B,(N)$

Mathematical Reviews subject classification: 26A45, 26A46, 26A48

Received by the editors May 27, 1997

*The author wishes to thank the referee for his suggestions which have led to an improved exposition. 
where $s_{F}$ is the saltus function of $F$ (clearly $s_{F}$ is a singular function).

Moreover we obtain

$$
V(F ;[a, x])=S_{F}(x)+(\mathcal{L}) \int_{a}^{x}\left|F^{\prime}(t)\right| d t,
$$

(for the definition of $S_{F}$ see Lemma 8).

The following decomposition theorem of Jordan is well known:

Theorem B (Jordan's decomposition theorem). ([6], p. 218). A function $F:[a, b] \rightarrow \mathbb{R}$ is $V B$ if and only if it is representable as the difference of two increasing functions.

In fact from the proof of this theorem it follows that if $F$ is $V B$ on $[a, b]$ then the functions $V_{F}(x):=V(F ;[a, x])$ and $G(x):=F(x)-V_{F}(x)$ are increasing. The question is which properties of $F$ will be preserved for $V_{F}$ and $G$ ? It is known that if $F$ is left, right or bilaterally continuous at a point $x \in[a, b]$ then so are $V_{F}$ and $G$ ([6], p. 223).

In the second part of this paper we show that if $F$ satisfies Lusin's condition $(N)$ then $V_{F}$ and $G$ also satisfy $(N)$.

\section{On Lebesgue's decomposition theorem}

We assume that the reader is familiar with the notions of $V B, A C$ and Lusin's condition $(N)$ (see [7], [6]). We denote by $\mathcal{C}$ the set of all continuous functions. If $F:[a, b] \rightarrow \mathbb{R}$ and $x_{o} \in[a, b)$ (resp. $\left.x_{o} \in(a, b]\right)$ then we denote by

$$
F\left(x_{o}+\right)=\lim _{\substack{x \rightarrow x_{o} \\ x>x_{o}}} F(x) \quad\left(\text { resp. } F\left(x_{o}-\right)=\lim _{\substack{x \rightarrow x_{o} \\ x<x_{o}}} F(x)\right)
$$

Lemma 1. (Theorem 1 of [6], p. 205). The sets of the discontinuity points of an increasing function $F:[a, b] \rightarrow \mathbb{R}$ is at most countable. If $x_{1}, x_{2}, x_{3}, \ldots$ are all of the interior discontinuity points then

$$
F(a+)-F(a)+\sum_{k}\left(F\left(x_{k}+\right)-F\left(x_{k}-\right)\right)+F(b)-F(b-) \leq F(b)-F(a) .
$$

Definition 1 (The saltus of an increasing function). ([6], p. 206). Let $F$ : $[a, b] \rightarrow \mathbb{R}$ be an increasing function. Let $A=\left\{a_{1}, a_{2}, \ldots\right\}$ be a countable subset of $[a, b]$ containing all the interior discontinuity points of $F$ (that this is possible follows by Lemma 1$)$. We define $s_{F}:[a, b] \rightarrow \mathbb{R}$ by $s_{F}(a)=0$ and for every $x \in(a, b]$,

$$
s_{F}(x)=F(a+)-F(a)+\sum_{t \in A \cap(a, x)}(F(t+)-F(t-))+F(x)-F(x-) .
$$


The function $s_{F}$ is called the saltus of $F$.

Let $A_{n}=\left\{a_{1}, a_{2}, \ldots, a_{n}\right\}$. We define $s_{F, n}(a)=0$ and for every $x \in(a, b]$,

$$
s_{F, n}(x)=F(a+)-F(a)+\sum_{t \in A_{n} \cap(a, x)}(F(t+)-F(t-))+F(x)-F(x-) .
$$

Remark 1. The functions $s_{F}$ and $s_{F, n}$ have the following properties:

(i) $s_{F}$ and $s_{F, n}$ are increasing on $[a, b]$, so $V B$ on $[a, b]$;

(ii) $s_{F, n}(x) \rightarrow s_{F}(x)$ for every $x \in[a, b]$;

(iii) $s_{F, n}$ is a constant on each component of the open set $(a, b) \backslash A_{n}$ (therefore $s_{F, n}$ is a step-function);

(iv) The function $F-s_{F}$ is increasing and continuous on $[a, b]$ (see Theorem 2 of [6], p. 206).

Lemma 2 (Sarkhel and Kar). ([8] or [2], [4]).

$V B \cap(N)$ is a linear space on $[a, b]$.

Lemma 3. Let $F:[a, b] \rightarrow \mathbb{R}$ be an increasing function. Then

$$
\begin{array}{cc}
s_{F}\left(x_{o}+\right)-s_{F}\left(x_{o}\right)=F\left(x_{o}+\right)-F\left(x_{o}\right), & x_{o} \in[a, b) \\
s_{F}\left(x_{o}\right)-s_{F}\left(x_{o}-\right)=F\left(x_{o}\right)-F\left(x_{o}-\right), & x_{o} \in(a, b] .
\end{array}
$$

Proof. Let $x_{o} \in[a, b)$ and $x>x_{o}$. Then

$s_{F}(x)=s_{F}\left(x_{o}\right)+F\left(x_{o}+\right)-F\left(x_{o}\right)+\sum_{t \in A \cap\left(x_{o}, x\right)}(F(t+)-F(t-))+F(x)-F(x-)$.

It follows that $s_{F}\left(x_{o}+\right)=\lim _{x \searrow x_{o}} s_{F}(x)=s_{F}\left(x_{o}\right)+F\left(x_{o}+\right)-F\left(x_{o}\right)$. Let $x_{o} \in(a, b]$ and $x<x_{o}$. Then

$s_{F}\left(x_{o}\right)=s_{F}(x)+F(x+)-F(x)+\sum_{t \in A \cap\left(x, x_{o}\right)}(F(t+)-F(t-))+F\left(x_{o}\right)-F\left(x_{o}-\right)$.

It follows that $s_{F}\left(x_{o}\right)=s_{F}\left(x_{o}-\right)+F\left(x_{o}\right)-F\left(x_{o}-\right)$.

Lemma 4. For $s_{F}$ defined above we have:

(i) $s_{F} \in(N)$ on $[a, b]$ and $s_{F}^{\prime}=0$ a.e. on $[a, b]$;

(ii) If $F \in(N)$ on $[a, b]$ then $F-s_{F} \in A C$ on $[a, b]$. 
Proof. (i) We have

$$
\begin{gathered}
s_{F}([a, b]) \subseteq\left[0, s_{F}(b)\right] \backslash\left(\left(0, s_{F}(a+)\right) \cup\right. \\
\left.\cup\left(\cup_{k=1}^{\infty}\left(\left(s_{F}\left(a_{k}-\right), s_{F}\left(a_{k}\right)\right) \cup\left(s_{F}\left(a_{k}\right), s_{F}\left(a_{k}+\right)\right)\right)\right) \cup\left(s_{F}(b-), s_{F}(b)\right)\right) .
\end{gathered}
$$

By Lemma 3 we have

$$
\begin{aligned}
& s_{F}(a+)=F(a+)-F(a), \\
& s_{F}\left(a_{k}+\right)-s_{F}\left(a_{k}-\right)=F\left(a_{k}+\right)-F\left(a_{k}-\right) \text { for each } k, \text { and } \\
& s_{F}(b)-s_{F}(b-)=F(b)-F(b-) .
\end{aligned}
$$

Since

$$
s_{F}(b)=F(a+)-F(a)+\sum_{k=1}^{\infty}\left(F\left(a_{k}+\right)-F\left(a_{k}-\right)\right)+F(b)-F(b-),
$$

it follows that $m\left(s_{F}([a, b])\right)=0$, hence $s_{F} \in(N)$ on $[a, b]$. Clearly $s_{F}$ is derivable a.e. on $[a, b]$. By Krzyzewski's Lemma (see [5] or [1], p. 70), we obtain that $s_{F}^{\prime}=0$ a.e. on $[a, b]$.

(ii) By Lemma 2 and Remark 1, (iv), it follows that $F-s_{F} \in V B \cap \mathcal{C} \cap(N)=$ $A C$ on $[a, b]$ (see the Banach-Zarecki Theorem [7], p. 227).

Remark 2. That $s_{F}^{\prime}=0$ a.e. in the proof of Lemma 4, (i), follows also from the following theorem of Fubini: If $F(x)=\sum_{n} F_{n}(x)$ is a convergent series of monotone nondecreasing functions on $[a, b]$ then $F^{\prime}(x)=\sum_{n} F_{n}^{\prime}(x)$ a.e. on $[a, b]$ ([7], p. 117), and Remark 1, (ii), (iii).

Definition 2. Let $\Delta: a=x_{o}<x_{1}<\ldots<x_{n}=b$ be a division of $[a, b]$, and let $g:[a, b] \rightarrow \mathbb{R}$. We denote

$$
V_{\Delta}(g ;[a, b])=\sum_{k=0}^{n-1}\left|g\left(x_{k+1}\right)-g\left(x_{k}\right)\right|
$$

and

$$
V(g ;[a, b])=\sup _{\Delta} V_{\Delta}(g ;[a, b]) \quad(\text { see }[6], \text { p. 215) }
$$

A division $\Delta_{1}: a=y_{o}<y_{1}<\ldots<y_{m}=b$ is said to be finer than $\Delta$ if $\left\{x_{o}, x_{1}, \ldots, x_{n}\right\} \subset\left\{y_{o}, y_{1}, \ldots, y_{m}\right\}$. Clearly $V_{\Delta}(g ;[a, b]) \leq V_{\Delta_{1}}(g ;[a, b])$. 
Lemma 5. Let $f:[a, b] \rightarrow \mathbb{R}, f \in \mathcal{C} \cap V B$, and let $h:[a, b] \rightarrow \mathbb{R}$ such that $h$ is constant on $(a, b)$. Then

$$
V(h ;[a, b])=|h(a+)-h(a)|+|h(b)-h(b-)|
$$

and

$$
V(f+h ;[a, b])=V(f ;[a, b])+V(h ;[a, b]) .
$$

Proof. The first part is obvious. We show the second one. Clearly

$$
V(f+h ;[a, b]) \leq V(f ;[a, b])+V(h ;[a, b]) .
$$

Let $\epsilon>0$. Since $f$ is continuous on $[a, b]$, there exists $\delta>0$ such that $|f(a)-f(x)|<\epsilon / 8$ if $x \in[a, a+\delta)$, and $|f(b)-f(y)|<\epsilon / 8$ if $y \in(b-\delta, b]$. Let

$$
\Delta: a=t_{o}<t_{1}<t_{2}<\ldots<t_{n}<t_{n+1}=b
$$

be a division of $[a, b]$ such that $V_{\Delta}(f ;[a, b])>V(f ;[a, b])-\epsilon / 2$. We may suppose without loss of generality that $t_{1} \in(a, a+\delta)$ and $t_{n} \in(b-\delta, b)$ (because if $\Delta_{1}$ is a finer division than $\Delta$ then $\left.V_{\Delta_{1}}(f ;[a, b]) \geq V_{\Delta}(f ;[a, b])\right)$. Then

$$
\begin{gathered}
V_{\Delta}(f+h ;[a, b])= \\
=\left|f\left(t_{1}\right)-f(a)+h\left(t_{1}\right)-h(a)\right|+\left|f\left(t_{2}\right)-f\left(t_{1}\right)\right|+\ldots+\left|f\left(t_{n}\right)-f\left(t_{n-1}\right)\right|+ \\
+\left|f(b)-f\left(t_{n}\right)+h(b)-h\left(t_{n}\right)\right| \geq\left|h\left(t_{1}\right)-h(a)\right|-2\left|f\left(t_{1}\right)-f(a)\right|+V_{\Delta}(f ;[a, b])- \\
-2\left|f(b)-f\left(t_{n}\right)\right|+\left|h(b)-h\left(t_{n}\right)\right|>|h(a+)-h(a)|-2 \frac{\epsilon}{8}+ \\
+V(f ;[a, b])-\frac{\epsilon}{2}-2 \frac{\epsilon}{8}+|h(b)-h(b-)|=V(f ;[a, b])+V(h ;[a, b])-\epsilon .
\end{gathered}
$$

Therefore $V(f+h ;[a, b]) \geq V(f ;[a, b])+V(h ;[a, b])$, hence $V(f+h ;[a, b])=$ $V(f ;[a, b])+V(h ;[a, b])$.

Corollary 1. Let $f:[a, b] \rightarrow \mathbb{R}, f \in \mathcal{C} \cap V B$, and let $g:[a, b] \rightarrow \mathbb{R}$ such that $g$ is constant on each of the intervals $\left(a, c_{1}\right),\left(c_{1}, c_{2}\right), \ldots,\left(c_{m}, b\right)$, where $a<c_{1}<c_{2}<\ldots<c_{m}<b$. Then

$$
\begin{gathered}
V(g ;[a, b])=|g(a+)-g(a)|+ \\
+\sum_{i=1}^{m}\left(\left|g\left(c_{i}\right)-g\left(c_{i}-\right)\right|+\left|g\left(c_{i}+\right)-g\left(c_{i}\right)\right|\right)+|g(b)-g(b-)|
\end{gathered}
$$

and

$$
V(f+g ;[a, b])=V(f ;[a, b])+V(g ;[a, b]) .
$$


Proof. We have

$$
\begin{gathered}
V(g ;[a, b])=V\left(g ;\left[a, c_{1}\right]\right)+\sum_{i=1}^{m-1} V\left(g ;\left[c_{i}, c_{i+1}\right]\right)+V\left(g ;\left[c_{m}, b\right]\right)= \\
|g(a+)-g(a)|+\left|g\left(c_{1}\right)-g\left(c_{1}-\right)\right|+\left|g\left(c_{1}+\right)-g\left(c_{1}\right)\right|+\left|g\left(c_{2}\right)-g\left(c_{2}-\right)\right|+\ldots+ \\
+\left|g\left(c_{m-1}+\right)-g\left(c_{m-1}\right)\right|+\left|g\left(c_{m}\right)-g\left(c_{m}-\right)\right|+\left|g\left(c_{m}+\right)-g\left(c_{m}\right)\right|+|g(b)-g(b-)|= \\
=|g(a+)-g(a)|+\sum_{i=1}^{m}\left(\left|g\left(c_{i}\right)-g\left(c_{i}-\right)\right|+\left|g\left(c_{i}+\right)-g\left(c_{i}\right)\right|\right)+|g(b)-g(b-)|
\end{gathered}
$$

(this equality was used before in [6], p. 231, but without proof). Now by Lemma 5

$$
\begin{gathered}
V(f+g ;[a, b])=V\left(f+g ;\left[a, c_{1}\right]\right)+\sum_{i=1}^{m-1} V\left(f+g ;\left[c_{i}, c_{i+1}\right]\right)+V\left(f+g ;\left[c_{m}, b\right]\right)= \\
=V\left(f ;\left[a, c_{1}\right]\right)+V\left(g ;\left[a, c_{1}\right]\right)+\sum_{i=1}^{m-1}\left(V\left(f ;\left[c_{i}, c_{i+1}\right]\right)+V\left(g ;\left[c_{i}, c_{i+1}\right]\right)\right)+ \\
+V\left(f ;\left[c_{m}, b\right]\right)+V\left(g ;\left[c_{m}, b\right]\right)=V(f ;[a, b])+V(g ;[a, b])
\end{gathered}
$$

Definition 3 (The saltus of a $V B$ function). ([6], p. 219).

Let $F:[a, b] \rightarrow \mathbb{R}, F \in V B$. Then we define $s_{F}:[a, b] \rightarrow \mathbb{R}$ by

$$
s_{F}(x)=s_{V_{F}}(x)-s_{G}(x),
$$

where $V_{F}(x)=V(F ;[a, x])$ and $G(x)=V_{F}(x)-F(x)$. Clearly $V_{F}$ and $G$ are increasing (see Theorem B).

Lemma 6. ([6], p. 233). Let $g_{n}, g:[a, b] \rightarrow \mathbb{R}$. If $\left\{g_{n}\right\}_{n}$ converges pointwise to $g$ on $[a, b]$ and there exists a positive number $\alpha$ such that $V\left(g_{n} ;[a, b]\right)<\alpha$, ( $\forall) n=1,2, \ldots$, then $V(g ;[a, b]) \leq \alpha$.

Lemma 7. Let $g, g_{n}:[a, b] \rightarrow \mathbb{R}$ such that $\left\{g_{n}\right\}_{n}$ converges pointwise to $g$ on $[a, b]$ and $V\left(g_{n} ;[a, b]\right) \nearrow \alpha$ for some positive number $\alpha$. If

$$
\liminf _{n} V\left(g_{n}-g ;[a, b]\right)=0
$$

then $V(g ;[a, b])=\alpha$. 
Proof. By Lemma 6 it follows that $V(g ;[a, b]) \leq \alpha$. We show the converse inequality. For $\epsilon>0$ let $n_{\epsilon}$ be a positive integer such that

$$
V\left(g_{n} ;[a, b]\right)>\alpha-\frac{\epsilon}{2},(\forall) n \geq n_{\epsilon} .
$$

For each $n \geq n_{\epsilon}$ we have

$$
\begin{aligned}
\alpha-\frac{\epsilon}{2}< & V\left(g_{n} ;[a, b]\right)=V\left(g-\left(g-g_{n}\right) ;[a, b]\right) \leq \\
& \leq V(g ;[a, b])+V\left(g-g_{n} ;[a, b]\right) .
\end{aligned}
$$

Since $\liminf \operatorname{in}_{n} V\left(g-g_{n} ;[a, b]\right)=0$, it follows that there exists a positive number $m \geq n_{\epsilon}$ such that $V\left(g-g_{m} ;[a, b]\right)<\epsilon / 2$. Therefore

$$
\alpha-\frac{\epsilon}{2} \leq V(g ;[a, b])+\frac{\epsilon}{2}
$$

so $\alpha \leq V(g ;[a, b])$. It follows that $\alpha=V(g ;[a, b])$.

Lemma 8. Let $F, G$, and $V_{F}$ be as in Definition 3.

(i) The set $A$ of the interior discontinuity points of $F$ is at most countable and contains the sets of interior discontinuity points of $V_{F}$ and $G$. Moreover, if $x \in A \cup\{a\}$ then there exists $F(x+)$ and for each $x \in A \cup\{b\}$ there exists $F(x-)$.

(ii) Let $A=\left\{a_{1}, a_{2}, a_{3}, \ldots\right\}$. Then $s_{F}(a)=0$ and for $x \in(a, b]$,

$$
s_{F}(x)=F(a+)-F(a)+\sum_{t \in(a, x) \cap A}(F(t+)-F(t-))+F(x)-F(x-) .
$$

(iii) If $A$ is infinite, let $s_{F, n}:[a, b] \rightarrow \mathbb{R}$ such that $s_{F, n}(a)=0$ and for $x \in(a, b]$,

$$
s_{F, n}(x)=F(a+)-F(a)+\sum_{t \in(a, x) \cap A_{n}}(F(t+)-F(t-))+F(x)-F(x-),
$$

where $A_{n}=\left\{a_{1}, a_{2}, \ldots, a_{n}\right\}$. Let

$$
r_{F, n}(x)=s_{F}(x)-s_{F, n}(x)=\sum_{t \in(a, x) \cap\left(A \backslash A_{n}\right)}(F(t+)-F(t-)) .
$$

We define $S_{F}:[a, b] \rightarrow \mathbb{R}$ by $S_{F}(a)=0$ and for every $x \in(a, b]$,

$$
S_{F}(x)=|F(a+)-F(a)|+
$$




$$
+\sum_{t \in A \cap(a, x)}(|F(t+)-F(t)|+|F(t)-F(t-)|)+|F(x)-F(x-)|
$$

(this series is convergent, see [6], p. 235). Then we have

1) $s_{F}$ is $V B$ on $[a, b]$;

2) $s_{F} \in(N)$ on $[a, b]$ and $s_{F}^{\prime}=0$ a.e. on $[a, b]$;

3) $F-s_{F}$ is continuous on $[a, b]$;

4) $s_{F, n}(x) \rightarrow s_{F}(x)$ for every $x \in[a, b]$;

5) $s_{F, n}$ is a constant on each component of the open set $(a, b) \backslash A_{n}$ (therefore $s_{F, n}$ is a step-function) and

$$
\begin{gathered}
V\left(s_{F, n} ;[a, b]\right)=|F(a+)-F(a)|+ \\
+\sum_{i=1}^{n}\left(\left|F\left(a_{i}\right)-F\left(a_{i}-\right)\right|+\left|F\left(a_{i}+\right)-F\left(a_{i}\right)\right|\right)+|F(b)-F(b-)| .
\end{gathered}
$$

6) If $n \rightarrow \infty$ then

$$
V\left(r_{F, n} ;[a, b]\right) \leq \sum_{t \in(a, b) \cap\left(A \backslash A_{n}\right)}(|F(t+)-F(t)|+|F(t)-F(t-)|) \rightarrow 0 ;
$$

7) $V\left(s_{F_{n}} ;[a, b]\right) \nearrow S_{F}(b)$;

8) $V\left(s_{F} ;[a, b]\right)=S_{F}(b)$.

Proof. (i) See [6] (Corollary 2, p. 219 and Theorem 1, p. 223).

(ii) See $[6]$ (p. 219).

(iii) 1) See the definition of $s_{F}$;

2) See Lemma 4, (i) and Lemma 2.

3) See [6] (p. 220).

4) This is obvious.

5) The first part is evident. We show the second part. For $i=1,2, \ldots, n$,

$$
\begin{aligned}
s_{F, n}(a+)-s_{F, n}(a) & =F(a+)-F(a) ; \\
s_{F, n}\left(a_{i}\right)-s_{F, n}\left(a_{i}-\right) & =F\left(a_{i}\right)-F\left(a_{i}-\right) ; \\
s_{F, n}\left(a_{i}+\right)-s_{F, n}\left(a_{i}\right) & =F\left(a_{i}+\right)-F\left(a_{i}\right) ; \\
s_{F, n}(b)-s_{F, n}(b-) & =F(b)-F(b-) .
\end{aligned}
$$

By Corollary 1 we obtain

$$
V\left(s_{F, n} ;[a, b]\right)=|F(a+)-F(a)|+
$$


ON THE DECOMPOSITION THEOREMS OF LEBESGUE AND JORDAN

$$
\sum_{i=1}^{n}\left(\left|F\left(a_{i}\right)-F\left(a_{i}-\right)\right|+\left|F\left(a_{i}+\right)-F\left(a_{i}\right)\right|\right)+|F(b)-F(b-)| .
$$

6) Let $a \leq x_{1}<x_{2} \leq b$. Then

$$
\begin{gathered}
r_{F, n}\left(x_{2}\right)-r_{F, n}\left(x_{1}\right)=\sum_{t \in\left[x_{1}, x_{2}\right) \cap\left(A \backslash A_{n}\right)}(F(t+)-F(t-)) \leq \\
\leq \sum_{t \in\left[x_{1}, x_{2}\right) \cap\left(A \backslash A_{n}\right)}(|F(t+)-F(t)|+|F(t)-F(t-)|),
\end{gathered}
$$

hence

$$
\begin{aligned}
& V\left(r_{F, n} ;[a, b]\right) \leq \sum_{t \in[a, b) \cap\left(A \backslash A_{n}\right)}(|F(t+)-F(t)|+|F(t)-F(t-)|)= \\
= & \sum_{t \in(a, b) \cap\left(A \backslash A_{n}\right)}(|F(t+)-F(t)|+|F(t)-F(t-)|) \rightarrow 0 \text { for } n \rightarrow \infty .
\end{aligned}
$$

7) This is evident.

8) See 4),6), 7) and Lemma 7.

Remark 3. $S_{F} \in(N)$ on $[a, b]$ and $S_{F}^{\prime}=0$ a.e. on $[a, b]$ (the proof is similar to that of Lemma 4 , (i)).

Lemma 9. Let $F:[a, b] \rightarrow \mathbb{R}, F \in V B \cap(N)$. Then $F-s_{F} \in A C$ on $[a, b]$.

Proof. By Remark 1, (iv), the functions $V_{F}-s_{V_{F}}$ and $G-s_{G}$ are continuous on $[a, b]$. Let

$$
\varphi=\left(V_{F}-s_{V_{F}}\right)-\left(G-s_{G}\right) .
$$

Then $\varphi$ is continuous on $[a, b]$. But

$$
\begin{gathered}
F=V_{F}-G=V_{F}-s_{V_{F}}+s_{V_{F}}-\left(G-s_{G}+s_{G}\right)= \\
=\varphi+\left(s_{V_{F}}-s_{G}\right)=\varphi+s_{F} .
\end{gathered}
$$

By Lemma 4, (i) the functions $s_{V_{F}}$ and $s_{G}$ belong to $V B \cap(N)$ on $[a, b]$, hence by Lemma $2, s_{F} \in V B \cap(N)$. Again by Lemma 2 , it follows that $\varphi=F-s_{F} \in V B \cap(N) \cap \mathcal{C}=A C$ on $[a, b]$ (see the Banach-Zarecki Theorem [7], p. 227).

Lemma 10. Let $F:[a, b] \rightarrow \mathbb{R}, F \in V B \cap(N)$ and let $F=H-h$, where $H$ and $h$ are some increasing functions given by Theorem $B$. Then $s_{F}=s_{H}-s_{h}$, hence $s_{F}$ does not depend on the choice of the functions $H$ and $h$ if $F \in V B \cap(N)$. 
Proof. By Lemma $9, F-s_{F} \in A C$ on $[a, b]$. But $F-\left(s_{H}-s_{h}\right)=\left(H-s_{H}\right)-$ $\left(h-s_{h}\right)$ is continuous (see Remark 1, (iv)). By Lemma 4, (i) and Remark 1, (i), $s_{H}, s_{h} \in V B \cap(N)$ on $[a, b]$. Since $F \in V B \cap(N)$, by Lemma 2 it follows that $F-\left(s_{H}-s_{h}\right) \in V B \cap(N)$ on $[a, b]$. From the Banach-Zarecki theorem we obtain that $F-\left(s_{H}-s_{h}\right) \in A C$ on $[a, b]$. Hence $s_{F}-\left(s_{H}-s_{h}\right) \in A C$ on $[a, b]$. By Lemma 4, (i), it follows that

$$
s_{F}^{\prime}=s_{H}^{\prime}=s_{h}^{\prime}=0 \text { a.e. on }[a, b] .
$$

Then $s_{F}-\left(s_{H}-s_{h}\right)$ is constant on $[a, b]$ (see for example Theorem 2 of [6], p. 246). Since $s_{F}(a)=s_{H}(a)=s_{h}(a)=0$, it follows that the above constant is zero, hence $s_{F}=s_{H}-s_{h}$ on $[a, b]$.

Theorem 1. Let $F:[a, b] \rightarrow \mathbb{R}, F \in V B$. Then $F \in(N)$ on $[a, b]$ iff

$$
F(x)-F(a)=s_{F}(x)+(\mathcal{L}) \int_{a}^{x} F^{\prime}(t) d t .
$$

Proof. " $\Rightarrow$ " Suppose that $F \in(N)$ on $[a, b]$ and let $\varphi=F-s_{F}$. Then $\varphi \in A C$ on $[a, b]$ (see Lemma 9), and by Lemma 4, (i) we have that $s_{F}^{\prime}=0$ a.e. on $[a, b]$. It follows that $\varphi^{\prime}=F^{\prime}$ a.e. on $[a, b]$. By Theorem 3 of [6] (p. 255), we obtain that

$$
\varphi(x)=\varphi(a)+(\mathcal{L}) \int_{a}^{x} \varphi^{\prime}(t) d t=F(a)+(\mathcal{L}) \int_{a}^{x} F^{\prime}(t) d t .
$$

Therefore we have (1).

" $\Leftarrow$ " Suppose that $(1)$ holds. The function $F(a)+(\mathcal{L}) \int_{a}^{x} F^{\prime}(t) d t$ is $A C$ on $[a, b]$ (see for example Theorem 1 of [6], p. 252), hence it is $V B \cap(N)$ on $[a, b]$ (for the $(N)$ part see Theorem 3 of [6], p. 249). By Lemma 8, (iii), 2), 1), it follows that $s_{F} \in V B \cap(N)$ on $[a, b]$. From (1) and Lemma 2, we obtain that $F(x)=s_{F}(x)+F(a)+(\mathcal{L}) \int_{a}^{x} F^{\prime}(t) d t$ is $V B \cap(N)$ on $[a, b]$.

\section{On Jordan's decomposition theorem}

Lemma 11. (Theorem 8 of $[6]$, p. 259). Let $f:[a, b] \rightarrow \overline{\mathbb{R}}$ be a summable function, and let

$$
F(x)=(\mathcal{L}) \int_{a}^{x} f(t) d t, \quad x \in[a, b] .
$$

Then

$$
V(F ;[a, x])=(\mathcal{L}) \int_{a}^{x}|f(t)| d t, \quad x \in[a, b] .
$$


Lemma 12. Let $f, F:[a, b] \rightarrow \mathbb{R}, F, f \in V B$. If $f$ is continuous on $[a, b]$ then

$$
V\left(f+s_{F} ;[a, b]\right)=V(f ;[a, b])+V\left(s_{F} ;[a, b]\right) .
$$

Particularly

$$
V(F ;[a, b])=V\left(s_{F} ;[a, b]\right)+V\left(F-s_{F} ;[a, b]\right) .
$$

Proof. Clearly $\left(f+s_{F, n}\right)(x) \rightarrow\left(f+s_{F}\right)(x)$ if $n \rightarrow \infty$ (see Lemma 8, (iii), 4)). By Corollary 1 and Lemma 8, (iii), 7), 8), we have

$$
\begin{gathered}
V\left(f+s_{F, n} ;[a, b]\right)=V(f ;[a, b])+V\left(s_{F, n} ;[a, b]\right) \nearrow V(f ;[a, b])+S_{F}(b)= \\
=V(f ;[a, b])+V\left(s_{F} ;[a, b]\right) .
\end{gathered}
$$

By Lemma 8, (iii), 6) and Lemma 7, it follows that

$$
V\left(f+s_{F} ;[a, b]\right)=V(f ;[a, b])+V\left(s_{F} ;[a, b]\right) .
$$

We show the second part. The function $f:=F-s_{F}$ is continuous and $V B$ on $[a, b]$ (see Lemma 8, (iii), 3)). Therefore

$$
V(F ;[a, b])=V\left(F-s_{F}+s_{F} ;[a, b]\right)=V\left(F-s_{F} ;[a, b]\right)+V\left(s_{F} ;[a, b]\right) .
$$

Theorem 2. Let $F:[a, b] \rightarrow \mathbb{R}, F \in V B \cap(N)$. Then

(i) $V_{F}(x)=S_{F}(x)+(\mathcal{L}) \int_{a}^{x}\left|F^{\prime}(t)\right| d t$;

(ii) $V_{F} \in(N)$ on $[a, b]$.

Proof. (i) From Theorem 1 we obtain

$$
\left(F-s_{F}\right)(x)=F(a)+(\mathcal{L}) \int_{a}^{x} F^{\prime}(t) d t .
$$

We have

$$
\begin{gathered}
V_{F}(x)=V(F ;[a, x])=V\left(s_{F} ;[a, x]\right)+V\left(F-s_{F} ;[a, x]\right)= \\
=S_{F}(x)+V\left(F-s_{F} ;[a, x]\right)=S_{F}(x)+V\left(F-s_{F}-F(a) ;[a, x]\right)= \\
=S_{F}(x)+(\mathcal{L}) \int_{a}^{x}\left|F^{\prime}(t)\right| d t
\end{gathered}
$$

(for the second equality see Lemma 12; for the third equality see Lemma 8 , (iii), 8); the fourth equality is obvious; the last equality follows by Lemma 11 and (2).

(ii) This follows since $S_{F} \in(N)$ (see Remark 3), by Lemma 2 and (i). 
Corollary 2. Let $F:[a, b] \rightarrow \mathbb{R}, F \in V B$. Then $F \in(N)$ on $[a, b]$ if and only if $V_{F} \in(N)$ on $[a, b]$.

Proof. " $\Rightarrow$ " See Theorem 2, (ii).

" $\Leftarrow$ " This follows as the implication " $\Leftarrow$ " in Lemma 6 of $[3]$.

Remark 4. Corollary 2 extends Lemma 6 of [3], since here $F$ is not supposed to be continuous on $[a, b]$.

Theorem 3 (A Jordan type theorem). Let $F:[a, b] \rightarrow \mathbb{R}, F \in V B \cap(N)$. Then $F$ is the difference of two increasing functions, each satisfying Lusin's condition $(N)$.

Proof. By Theorem 2, (ii) we have that $V_{F} \in(N)$ on $[a, b]$, and by Lemma 2 , the function $G=V_{F}-F \in(N)$.

\section{References}

[1] V. Ene, Real functions - current topics, Lect. Notes in Math., vol. 1603, Springer-Verlag, 1995.

[2] V. Ene, On Borel measurable functions that are $V B G$ and $(N)$, Real Analysis Exchange 22 (1996-1997), no. 2, 688-695.

[3] V. Ene, An elementary proof of the Banach-Zarecki theorem, Real Analysis Exchange, 23 (1997/1998), no. 1.

[4] V. Ene, Lusin's condition $(N)$ and Foran's condition $(M)$ are equivalent for Borel functions that are VBG on a Borel set, (submitted).

[5] K. Krzyzewski, On change of variable in the Denjoy-Perron integral (I),(II), Coll. Math. 9 (1962), 99-104 and 317-323.

[6] I. P. Natanson, Theory of functions of a real variable, 2nd. rev. ed., Ungar, New York, 1961.

[7] S. Saks, Theory of the integral, 2nd. rev. ed., vol. PWN, Monografie Matematyczne, Warsaw, 1937.

[8] D. N. Sarkhel and B. Kar, (PVB) functions and integration, J. Austral. Math. Soc. (Series A) 36 (1984), 335-353. 